\title{
Diseño y Longevidad: consideraciones para el desarrollo de proyectos para adultos mayores que involucran tecnologías no familiares
}

\section{Design and Longevity: considerations for the development of projects for older adults that involve non-familiar technologies}

\author{
Dra. Georgina Durán Quezada. Universidad Iberoamericana Ciudad de México \\ georgina.duran@ibero.mx
}

\section{Dra. Vera Maria Marsicano Damazio. Pontifícia Universidade Católica do Rio de Janeiro}

vdamazio@puc-rio.br

\section{Resumen}

Hace mucho que la humanidad camina lado a lado de la tecnología, encantándose e inquietándose con ella en mayor o menor grado. Sin embargo, los avances tecnológicos de las últimas décadas transforman el planeta, sus sociedades y habitantes de forma acelerada y sin precedentes. Entre sus efectos, está el irreversible fenómeno de envejecimiento y longevidad de la población en la casi totalidad de los países. Estamos envejeciendo, viviendo más y rodeados de productos y servicios que impactan todas las esferas de nuestra existencia. Además de creciente, se trata del más heterogéneo de todos los grupos de edad, el público mayor abarca desde adultos con 60 años hasta los centenarios. En ese sentido, requiere atención especial para que sus variadas demandas sean reconocidas y atendidas a satisfacción. Este artículo presenta resultados del estudio etnográfico sobre la relación entre los adultos mayores y nuevas tecnologías, así como directrices para el desarrollo de proyectos para el público senior que involucren tecnologías no familiares.

Palabras clave: Envejecimiento, Transformaciones tecnológicas, Diseño

\section{Resumo}

Há muito, a humanidade caminha lado a lado à tecnologia e vem se encantando e se inquietando com ela em maior ou menor grau. Os avanços tecnológicos das últimas décadas, contudo, vêm transformando o planeta, suas sociedades e seus habitantes de forma acelerada e sem precedentes. Dentre seus efeitos, está o irreversível fenômeno de envelhecimento e longevidade da população na quase totalidade dos países. Estamos envelhecendo, vivendo mais e cercados de produtos e serviços que impactam todas as esferas de nossa existência. Além de crescente, o público sênior abrange desde adultos com 60 anos até os centenários e trata-se do mais heterogêneo de todos os grupos etários. Nesse sentido, requer atenção especial para que suas variadas demandas sejam reconhecidas e atendidas a contento. Este artigo apresenta resultados de estudo etnográfico sobre a relação entre adultos idosos e novas tecnologias e diretrizes para o desenvolvimento de projetos para o público sênior que envolvam tecnologias não familiares.

Palavras-chave: Envelhecimento, Transformações tecnológicas, Design 


\section{Abstract}

Humanity has long been walking side by side with technology and has been enchanting and frightening by it to a greater or lesser extent. The technological advances of the last decades, however, have been transforming the planet, its societies and its inhabitants in an accelerated and unprecedented way. Among its effects is the irreversible phenomenon of population aging and population longevity in almost all countries. We are aging, living longer and surrounded by products and services that impact all spheres of our existence. In addition to growing, the senior public ranges from 60-year-olds to centenarians and is the most heterogeneous of all age groups. In this sense, it requires special attention so that its varied demands are well acknowledged and satisfied. This article presents results of an ethnographic study on the relationship between older adults and new technologies and guidelines for the development of projects for the senior public that involve unfamiliar technologies.

Keywords: Ageing, Technological Transformations, Design

\section{Introducción}

\section{Adultos mayores y diversidad}

El envejecimiento humano es un proceso que tiene implicaciones sociales, económicas, laborales y familiares, afectando mente, espíritu y ambiente, así como la vida social, económica y cultural.

La vejez, por su parte, es resultado de un proceso dinámico en el que existe desarrollo (ganancias), estabilidad y declive (pérdidas) (FERNÁNDEZ-BALLESTEROS, 2011). La figura 1, ejemplifica diferentes habilidades y/o áreas sobre las que se observan modificaciones a lo largo del tiempo, algunas permanecen, otras presentan declives (memoria de trabajo, coordinación, fuerza), o en dado caso, aumentos (percepción de bienestar, sentido de coherencia, red familiar).

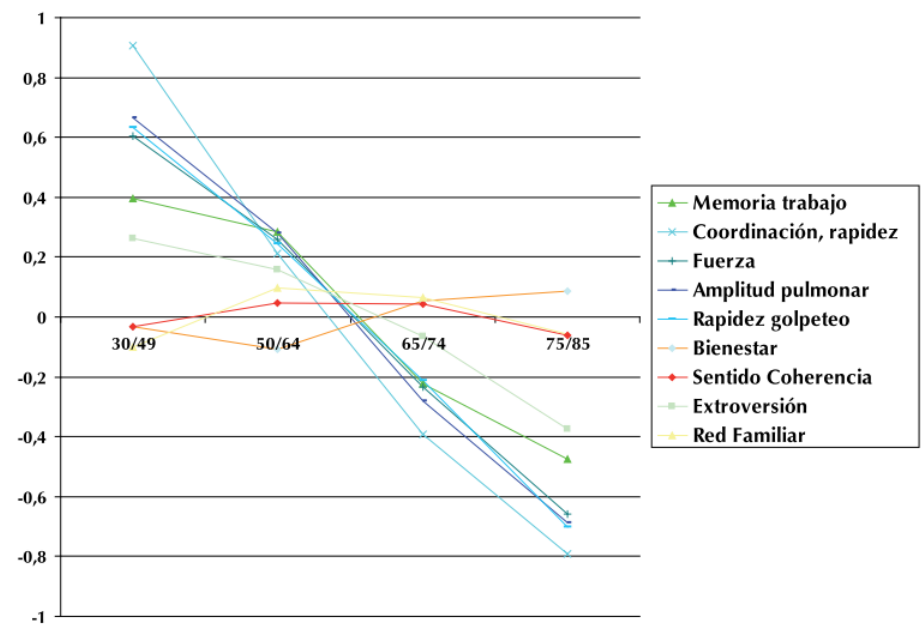

Figura 1. Estabilidad y declive en características físicas, psicológicas y sociales (FERNÁNDEZ-BALLESTEROS, 2011, p. 118) 
De acuerdo con Fernández-Ballesteros (2004a, p. 48), el envejecimiento puede reflejarse de tres maneras: (1) normal, (2) patológico -con discapacidades o pérdidas a consecuencia de enfermedades o accidentes- , o (3) con éxito. En las tres posibilidades, las condiciones físicas, psicológicas y cognitivas de las personas pueden modificarse de diferentes maneras.

Con la edad, es posible que algunos de estos cambios se presenten:

1. Condiciones Físicas: Pérdida de audición, visión y movilidad. Cardiopatías, accidentes cerebrovasculares, enfermedades respiratorias, cáncer y demencia (OMS, 2015). Problemas con el equilibrio, articulaciones y superficies óseas, disminución de la estatura, cambios en el metabolismo (KROEMER, 2006).

2. Condiciones Psicológicas: En esta última etapa de la vida, se pueden vivir situaciones difíciles; los duelos, los cambios de roles o las enfermedades, pueden traer depresión y sufrimiento. Sin embargo, no ocurre así en todos los casos. Por el contrario, en estos años puede existir una mayor complejidad y riqueza emocional, así como una mayor madurez afectiva.

3. Condiciones Cognitivas: Envejecer, en general, produce un funcionamiento cognitivo menos eficiente; la memoria de trabajo (usada para recordar nuevos nombres y teléfonos) y la memoria episódica (usada para recordar cuándo se vio a alguien por última vez), se ven afectadas, aunque no tanto la semántica (usada para recordar a los héroes de la independencia) y la procedimental (usada para conducir un automóvil). La inteligencia biológica o fluida (relacionada a la velocidad perceptiva, fluidez verbal, razonamiento, aptitud espacial), declina, mientras que la inteligencia cultural o cristalizada (relacionada a las aptitudes de información, comprensión, vocabulario), se mantiene constante (FERNÁNDEZ-BALLESTEROS, 2004b).

Resulta que la "vivencia de sentirse 'viejo' es propia de cada individuo y sus circunstancias" (PICARDI, 2009, p. 8). Así pues, desarrollar proyectos para el público senior es un reto para el diseñador, ya que este grupo no encaja en una sola definición. Delimitar sus características es tarea difícil, ya que esta es una población sumamente diversa, que incluye desde sexagenarios a centenarios activos o incapacitados por diferentes condiciones físicas y/o cognitivas. Al desarrollar proyectos centrados en los adultos mayores, es necesario reconocer tanto las ganancias como las pérdidas de la vejez, pero, al mismo tiempo, dar relieve y enfocar sus fortalezas (sabiduría, experiencia o conocimientos acumulados).

\section{El impacto del desarrollo de las nuevas tecnologías en las personas mayores}

En diferentes épocas, la humanidad se ha enfrentado a las transformaciones tecnológicas con una mezcla entre asombro y recelo. Ha sido muy común que, frente a los cambios, la percepción, es de que nunca la sociedad había avanzado tanto. Por otro lado, las tecnologías que ahora se ven sencillas y de uso común, tuvieron en el pasado que ser aprendidas, y muy posiblemente maravillaban, pero también confundieron y asustaron a muchas personas.

A principio del siglo XX, la entrada de los automóviles, el gas, la electricidad y el agua corriente en las ciudades era algo innovador, pero también, muy extraño para algunos. Por ejemplificar, es posible mencionar que a mediados del siglo pasado, mucha gente encontraba 
arriesgado y perturbador subir a un elevador sin ascensorista, tal y como ocurre ahora con los automóviles autónomos (HENN, 2015).

Los cambios tecnológicos, sobre todo los que son disruptivos, impactan en la vida diaria de tal forma que no solamente afectan nuestra manera de trabajar, convivir o entretenernos. Tal como explica McLuhan (1998, p. 16): "ya no sentimos del mismo modo, ni continúan siendo los mismos nuestros ojos, nuestros oídos, nuestros restantes sentidos".

Simone de Beauvoir (1986, p. 459) reconocía que los adultos mayores, como consumidores, eran beneficiarios del progreso técnico, sin necesariamente ser incomodados por él. De hecho, en algunos casos, los ancianos resultaban más receptivos a los avances que los hombres de menor edad (50 a 60 años), los que se sentían en conflicto por sus intereses económicos e ideológicos, o amenazados por los jóvenes que manejaban mejor las nuevas máquinas.

Los adultos mayores han sido percibidos (por la sociedad y por ellos mismos), desde hace ya tiempo, como personas que quedan atrás del uso de las innovaciones tecnológicas. En el siglo XVIII, Jonathan Swift, escritor y autor de "Los viajes de Gulliver", veía un mundo cambiante, en el que el viejo se estancaba y no era capaz de "acompañar su evolución" quedando "rezagado, solo, aislado, privado de todo lo que se aleja de él" y vedado de comunicarse con las generaciones más jóvenes (DE BEAUVOIR, 1986, p. 229).

Cabe destacar que también es característica del grupo de adultos mayores, la diversidad en la adopción de nuevas tecnologías. Mientras que muchos intentan mantenerse actualizados y disfrutan de lo "nuevo", otros nunca han estado en línea (KHAN, 2013, p. 14).

El diseño ha tenido un papel crucial en la adopción de nuevas tecnologías. Por mencionar un ejemplo, la mejora en las interfaces, usabilidad y simplicidad en los teléfonos móviles puede ser definitoria en la cantidad de adultos mayores que quieran utilizarlos en lugar de las opciones con las que ya cuentan. A pesar de que la tendencia es a mejorar, aún hay muchas áreas de oportunidad. El desarrollo de productos y servicios, tanto los que contienen, como los que no contienen elementos tecnológicos, tienden a ser pensados para un grupo de población joven y buena capacidad funcional, por lo que resulta ajena para el día a día y capacidades de los mayores.

\section{Método para el desarrollo de propuestas de diseño para adultos mayores}

El diseño ha demostrado ser eficiente en la búsqueda de solución a desafíos sociales complejos tal y como lo es el envejecimiento poblacional.

El desarrollo de productos y servicios para adultos mayores implica una aproximación empática para el entendimiento de sus necesidades, deseos, entorno y contexto. Realizar la investigación de primera mano, con la consigna de procurar entender y tratar de ponerse en el lugar del otro, se vuelve una experiencia invaluable para el proceso de diseño.

Son varias las maneras con las cuales podemos obtener la información y conocimientos necesarios para diseñar. Es innegable la utilidad de los métodos de investigación documentales y cuantitativos, sin embargo, considerando las múltiples diferencias que presentan los adultos mayores, es fundamental el uso de métodos cualitativos para entender sus características y 
singularidades, así como la relación que ellos tienen con los productos y servicios, especialmente si nos referimos a tecnologías que les son ajenas.

La etnografía, ayuda a entender lo que ocurre y las razones por las que ocurren. Sus métodos, al ser utilizados en el diseño se caracterizan por la búsqueda del entendimiento de las personas y su realidad, fundamentando el proceso de desenvolvimiento de soluciones proyectuales. El diseñador participa durante el mayor tiempo posible, dadas las restricciones del proyecto: escucha, observa, pregunta y convive con la finalidad de arrojar luz sobre el tema que deberá solucionar. Los métodos etnográficos implican el involucrarse de manera abierta, atenta, humilde y dispuesta.

\section{Conviviendo con los adultos mayores}

La investigación etnográfica de este trabajo inició en la Casa de Convivencia e Lazer Maria Haydée (CCLMH) en Río de Janeiro en 2013 y continuó en 2014-2015 en las instalaciones de Abue's Club (AC) ${ }^{1}$ en la Ciudad de México. Ambas instituciones reciben adultos mayores con interés en realizar actividades de ocio y aprendizaje, así como en socializar. Tanto una como otra, atienden a las personas solamente durante el día y no tienen servicios de permanencia y habitaciones.

La CCLMH es parte del programa del ayuntamiento de la Ciudad de Rio de Janeiro para las personas mayores de 60 años, ofreciendo actividades como manualidades, clases de idioma, teatro o yoga, además de organizar reuniones o concursos. Los asistentes son en promedio, adultos mayores a los 65, con capacidades funcionales suficientes para poder realizar las actividades por ellos mismos. Aunque de acuerdo con los trabajadores sociales, algunos presentan síntomas de depresión o demencia senil, ellos se perciben como fuertes y saludables para su edad.

AC es un centro privado, en el que se realizan actividades como taichí, manualidades, rutinas de ejercicios físicos, sistemas de estimulación cognitivas y dinámicas para reafirmar la trascendencia y el sentido de la vida. Sus asistentes son en promedio adultos mayores a los 70 años y presentan diferentes grados de capacidad funcional. Aunque casi todos pueden realizar actividades de manera independiente, algunos presentan fallos en la memoria, dificultad auditiva o pequeños problemas físicos, que los llevan a usar el bastón o a tener tremores en las manos.

La investigación en ambos centros tuvo como objetivo analizar la relación de los adultos mayores con las nuevas tecnologías y cómo ellas impactan sus vidas cotidianas. Se propuso una experiencia de aprendizaje con adultos mayores a 70 años; es decir, se les ofreció enseñarles cuestiones básicas de informática, ya sea en computadora de escritorio o tableta, lo cual sirvió como pretexto para poder observar y hablar sobre: (1) el impacto que los sistemas digitales tienen en su vida (2) el tipo de interacción que se da con estos sistemas y (3) las razones, motivaciones o frustraciones asociadas con integrarse a una nueva tecnología.

El contacto con los trabajadores sociales de la CCLMH se dio por medio de una investigadora de la Pontifícia Universidade Católica do Rio de Janeiro (PUC-Rio) y los primeros acercamientos

${ }^{1}$ El nombre de la institución fue cambiado 
a los frecuentadores se dieron en sus instalaciones con la intención de conocerlos y tener momentos de inmersión en sus propias clases. Se identificó un grupo tanto de mayores como de trabajadores sociales, sumamente animados y dispuestos a intentar actividades que involucran el uso de computadoras. En un principio, fue realizado un sondeo sobre el tipo de actividades que querían desarrollar, la cantidad de personas que se integrarían o las razones para aprender a usar la computadora. Se habló también con las personas encargadas de la CCLMH, para compartir las intenciones del estudio y para solicitar ayuda (que fue otorgada con gran presteza).

Las clases de cómputo para los adultos mayores frecuentadores de la CCLMH se planearon para ser realizadas en las instalaciones de PUC-Rio, quienes asignaron un salón de clases regular, con computadoras PC y sistema operativo Windows. La Coordinación Central de Extensión (CCE) de la Universidad, facilitó la instalación de los programas y el material para las clases.

Después de haber identificado los deseos y demandas de los participantes, se elaboraron los contenidos de las seis sesiones que fueron realizadas en el transcurso de dos semanas. Fueron demostrados de manera básica, las búsquedas de Google, las videoconferencias en Skype y Facebook. Se pidió a los asistentes que se inscribieran a los temas que les parecieron interesantes. Cada uno de los tres bloques tuvo asignado una profesora $y$ al menos tres observadoras/asistentes/ayudantes.

Aunque varió un poco según los días, en promedio, se tuvo un grupo de 10 personas, de los cuales solo asistió un hombre. El promedio de edad fue de 75 años y el nivel socioeconómico, medio alto $(\mathrm{C}+\mathrm{y} \mathrm{B})$.

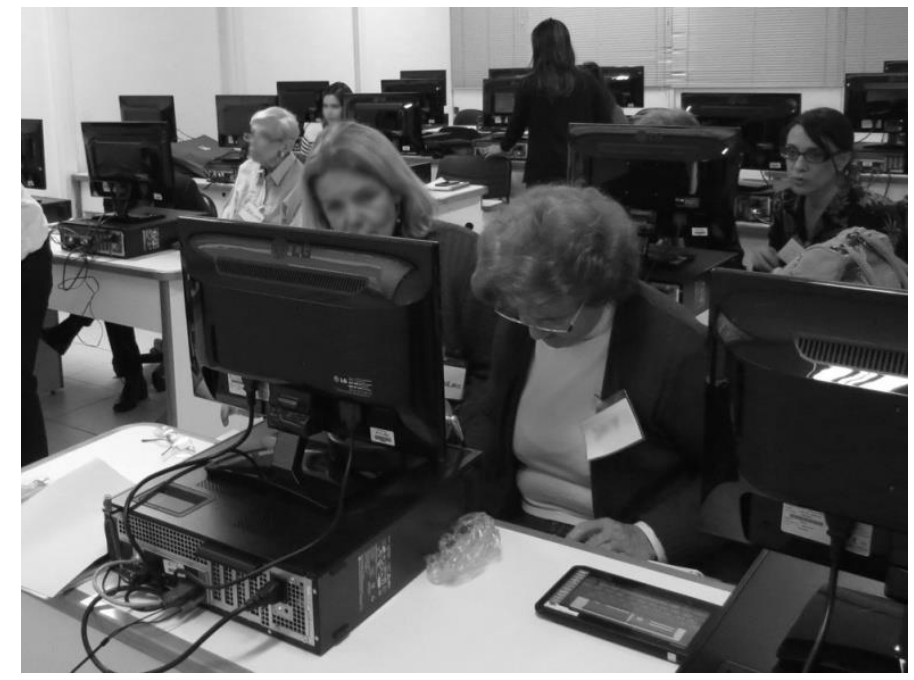

Figura 1 - Adultos mayores en clase de computadora en Rio de Janeiro

La actividad realizada en Rio de Janeiro fue replicada en la Ciudad de México con el mismo objetivo de analizar las relaciones de los adultos mayores con las nuevas tecnologías, modificando algunas de las condiciones, como la cantidad de asistentes. No se tenía relación previa con el Abue's Club (AC), por lo que se realizó un acercamiento con la Directora General, quien comentó que, aunque nunca habían ofrecido servicios de cómputo, algunas personas habían mostrado interés. Se acordó un día en la semana para comenzar con las sesiones semanales con no más de cuatro adultos mayores por encuentro, siendo los empleados de AC los encargados de invitar a las personas que asistirían. 
El ambiente asignado para la actividad era acogedor y tenía una mesa redonda en donde en cada sesión se instalaban las computadoras portátiles de AC o las tabletas que cada asistente traía desde su casa.

Para los adultos mayores de AC, se realizó una actividad por más tiempo, con formato más libre, en un ambiente más informal, en la que las personas pudieran llevar sus equipos y sus propias solicitudes en cada encuentro. Se pensó que se cubrieran temas básicos como se hizo con los frecuentadores del CCLMH en Río de Janeiro (videoconferencias, búsquedas, Facebook, algunos juegos). La flexibilidad del esquema hizo que, con el tiempo, cada uno decidiera lo que quería experimentar en el momento, por lo que era posible que uno de los asistentes estuviera en Pinterest, mientras que otro revisaba su correo.

La investigación etnográfica en AC duró un año, en la que se tuvieron sesiones con duración de hora y media, una vez a la semana. Durante ese período, seis personas con edad media de 85 años asistieron los encuentros con regularidad. Al final se realizó una entrevista individual a los tres integrantes más asiduos.

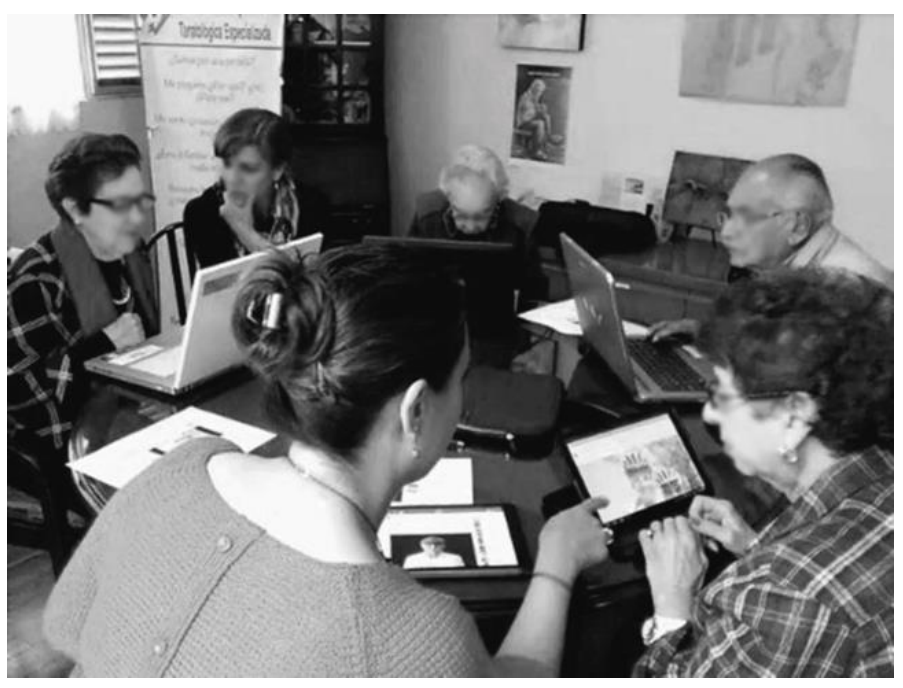

Figura 2 - Sesión en Abue’s Club

A lo largo del trabajo de campo se reunió material fotográfico, transcripciones de las entrevistas y clases, así como una bitácora con las anotaciones resultantes de las observaciones realizadas durante las sesiones. El análisis mantuvo la mirada sobre los factores sociales, afectivos y de motivación relacionados con la adopción de nuevas tecnologías, así como las cuestiones que responden a las capacidades funcionales (características físicas y cognitivas) que podrían influenciar el deseo o facilidad de uso. A continuación, se enumeran algunos de los hallazgos más representativos de la investigación sobre la relación entre los adultos mayores y nuevas tecnologías, organizados por grandes temas:

\section{Sociabilidad y Familia}

- El apoyo recibido de los integrantes de la familia, y particularmente de hijos y nietos, tienen gran influencia en la adopción de las nuevas tecnologías;

- Varios compartieron su sentir de que el no hacer uso de las redes sociales o la computadora obstaculiza la participación en las conversaciones con la familia y otras personas; 
- La interacción social y la comunicación con familiares - y sobre todo nietos - que viven lejos constituyen las principales razones para comenzar a utilizar algún sistema de cómputo.

\section{Motivaciones}

Diferentes motivaciones pueden influir en la adopción de nuevas tecnologías, como:

- Aprender cosas nuevas, así como asistir a actividades diferentes;

- Buscar temas para posteriores conversaciones con los amigos y familia;

- Mantenerse actualizados y que los demás los perciban de esa manera.

La motivación puede darse también a partir del establecimiento de la relación de la tecnología y las posibilidades ofrecidas de la computadora con:

- Deseos, necesidades, experiencias y expectativas particulares. En el caso de adquisición de nuevos conocimientos y contenidos, era crucial establecer conexión y sentido con lo que el adulto mayor necesitaba y estaba viviendo, para que pudieran ser aprendidos, recordados y posteriormente aplicados;

- Actuación, competencias, tareas y hechos profesionales realizados en la juventud y madurez, como revisar el correo o usar un procesador de palabras;

- Aficiones, pasatiempos, hobbies, actividades de ocio y recreativas, como las películas, libros y los juegos que fueron preferidos durante la juventud y la madurez, así como aquellos que se han ido sumando durante su vejez.

\section{Emociones}

- El proceso de adoptar una tecnología ajena o el uso de la computadora, tabletas o teléfonos móviles generaran tanto emociones agradables (como el logro y el asombro), como desagradables (frustración y fracaso);

- Se notó cierta inseguridad y dificultad para reponerse de los errores. Al ocurrir algo inesperado (como la aparición de un anuncio pop-up), se tuvo la tendencia aquedarse pasivos y no realizar nada más y a esperar a que alguien más fuera personalmente a ayudarlos. En algunos casos, se interpretaron los mensajes de la computadora como que si ellos hubieran cometido un error;

- El no entender todo lo que ocurría en la pantalla, generó angustia y estrés;

- Fue posible distinguir un sentimiento de ignorancia, de exclusión y de analfabetismo por no poder utilizar la computadora. La dificultad de aprender una nueva tecnología los hacía sentirse incapaces y hasta disminuidos y humillados;

- El olvidar cómo se utiliza (no solo de sesión a sesión), también generó frustración y los hizo sentirse incapaces.

- Por otro lado, aprender a usar el computador los ayudó a sentir una renovación ya dejar de "sentirse envejecidos";

- Fue reportado un miedo y preocupación recurrente y muy grande a los "peligros de Internet", como el de que sus recursos económicos sean robados; 
- Había también, una preocupación constante sobre el estado de sus archivos, como, por ejemplo, la disponibilidad y ubicación de sus fotografías.

\section{Capacidad Funcional}

\section{a. física}

- Para aquellos que presentan tremores, los teclados y las pantallas táctiles resultan complicados;

- La baja visión y/o baja audición causaron cierta dificultad, pero los asistentes pudieron resolverlo;

- Las repercusiones más fuertes de presentar disminución de las capacidades físicas son: el tiempo empleado en el uso y la frustración por el trabajo extra implicado;

- Aunque no negaban las disminuciones en sus condiciones físicas o cognitivas, cuando se les preguntaba, no hacían referencia a las dificultades que estaban teniendo debido a estas.

b. cognitiva

- La memoria es un gran factor por considerar. Expresaron preocupación por olvidar las instrucciones, reconocían que su memoria les fallaba y creían que no podrían repetir lo que habían aprendido. Hacer anotaciones por escrito en un medio físico como cuadernos, les daba la seguridad de que las instrucciones podrían ser consultadas posteriormente.

- Necesitaban y solicitaban repetir una y otra vez lo aprendido con la finalidad de poderse acordar después.

Adicional a las observaciones anteriores, se describen también a continuación, factores que están relacionados con el contexto y ambiente:

\section{Uso de los programas y equipos}

- Los procedimientos que involucran registros y sus correspondientes accesos (usuarios y contraseñas), son particularmente complicados para estos usuarios. La creación de un registro, por ejemplo, es un proceso complejo que no necesariamente entendían. Los usuarios y contraseñas necesitaban ser escritos y revisados continuamente.

- El uso de equipos de escritorio demostró la complejidad de interactuar con la computadora a través de 3 dispositivos (teclado, ratón y monitor).

- Aunque el teclado y su distribución no son una tecnología nueva, lo fue para ciertas personas que no tuvieron la necesidad de utilizar uno, durante sus años productivos. En este caso, se volvió una cuestión incremental, ya que las letras les resultaban muy difíciles para encontrar.

- La interacción con sistemas y programas poco consistentes, como por ejemplo en el uso de un clic o doble clic, resultan confusos, extraños y no les hacia sentido.

\section{La actividad de aprendizaje}

- Hubo una tendencia entre los asistentes a ser muy demandantes con respecto a tener la presencia de alguien que los auxiliara de manera particular e individual;

- Una de las motivaciones para la asistencia a cursos y actividades, no solo en este caso, es la posibilidad de socializar, y el pretexto para salir de su casa; 
- Hubo una demanda casi generalizada de tener material en papel (instrucciones, manuales o apuntes);

- Algunas de las palabras de uso común para quien utiliza de manera cotidiana las redes sociales y los sistemas de cómputo, no hacen ningún sentido para los mayores a quienes esta tecnología les es ajena.

\section{Consideraciones finales}

La estrategia de examinar la relación entre los adultos mayores y la tecnología no familiar a partir de clases prácticas sobre el uso del ordenador y sus programas resultó efectiva al ser un espacio ideal para conversar y observar las cuestiones propuestas por la investigación y se reveló un método eficiente, espontáneo y productivo.

El triple papel representado (diseñadora, investigadora, profesora), ocasionó el que hubiera que voltear la mirada de diferentes maneras y para diferentes cuestiones: lo que aprendían, los comentarios, las distracciones, los temas, o el monitoreo de los tiempos. A ratos el foco de la investigación parecía perderse. Sin embargo, durante todo el tiempo hubo una aproximación empática, escuchando atentamente, procurando entender los sentimientos positivos y negativos que surgían durante las actividades y sus razones. El modelo de investigación, aunque largo para ser utilizado en un proyecto de diseño "regular", resultó ser provechoso y extremadamente adecuado y necesario como instrumento de estudios centrados en el individuo en general y orientados a la relación de uso entre las personas y productos y servicios de cualquier naturaleza.

Se reconoce la estrategia de impartir clases como un método, al igual que otros de los métodos etnográficos, que permite al diseñador conectarse con la comunidad o las personas, obteniendo un conocimiento profundo, para proponer soluciones que tengan utilidad y significado.

Es importante resaltar que los años en los que fue realizada esta investigación, resultaron un momento muy particular. Actualmente se ven más adultos mayores utilizando los dispositivos móviles, lo que ayuda a minimizar el sentimiento de sentirse arcaico. Algunas cosas que eran de gran interés, como el manejo de fotografías, se han simplificado con el avance de la tecnología y la mayor capacidad de almacenamiento de estos.

En todas las épocas se han dado transformaciones tecnológicas, aunque en siglos anteriores, menos aceleradas. Siempre ha habido personas que abrazan los cambios con entusiasmo, quienes se asustan y sospechan, o quienes simplemente prefieren quedarse con lo que les es familiar. Lo cotidiano para los jóvenes, puede resultarles ajeno y difícil, pero ellos suelen tener más vigor y facilidad para enfrentar lo nuevo y desconocido. Desafortunadamente, no podemos decir lo mismo de los adultos mayores ya que es recurrente que, poco a poco, pero de manera sostenida, vayan dejando de estar al día y acaben sintiendo una extrañeza de lo que les rodea. Aunque no aplica para todos, los adultos mayores, tienden a resentir estos cambios tecnológicos más que el resto de la población.

Es importante reconocer que no hay grupo de edad más creciente y diverso que el de los adultos mayores. Este vivido y variado público, del que ya somos o seremos parte un día, necesita ser conocido y atendido empáticamente, presentando un campo de oportunidades sin igual para los diseñadores. 


\section{Referencias}

DE BEAUVOIR, Simone. La Vejez. 2a reimpre ed. México: Editorial Hermes, 1986.

DURAN QUEZADA, Georgina. Las transformaciones tecnológicas y los adultos mayores : Consideraciones para el desarrollo de propuestas de Diseño que involucran tecnologías no familiares. 2017. 210 f. Pontificia Universidad Católica de Rio de Janeiro, $2017 . \quad$ Disponível em: <http://www2.dbd.pucrio.br/pergamum/tesesabertas/1313954_2017_completo.pdf>.

FERNANDEZ-BALLESTEROS, Rocío. Gerontología Social. Madrid: Pirámide, 2004.

FERNÁNDEZ-BALLESTEROS, Rocío. La psicología de la vejez. Encuentros Multidisciplinarios, v. 16, 2004.

FERNÁNDEZ-BALLESTEROS, Rocío. POSIBILIDADES Y LIMITACIONES DE LA EDAD. Libro blanco de las personas mayores y del envejecimiento. [S.l: s.n.], 2011. p. 113150.

HENN, Steve. Remembering When Driverless Elevators Drew Skepticism (Interview whith LEE GRAY). Disponível <http://www.npr.org/2015/07/31/427990392/remembering-when-driverless-elevators-drewskepticism>. Acesso em: 22 jul 2016.

KHAN, Halima. Five Hours a Day: Systemic Innovation por an Ageing Population. . London, UK: NESTA. , 2013

KROEMER, K. H. E. Designing for Older People. Ergonomics in Design: The Quarterly of Human Factors Applications, v. 14, n. 4, p. 25-31, 1 Out 2006. Disponível em: <http://erg.sagepub.com/lookup/doi/10.1177/106480460601400407>. Acesso em: 9 ago 2013.

MCLUHAN, Marshall. La Galaxia Gutenberg: Genesis del "Homo Typographicus". Barcelona: Círculo de Lectores, 1998.

OMS. Informe Mundial sobre el Envejecimiento y la Salud. USA: OMS, 2015. Disponível em: <http://www.who.int/ageing/publications/world-report-2015/es/>.

ORGANIZACIÓN MUNDIAL DE LA SALUD. Envejecimiento y Salud. Disponível em: <http://www.who.int/es/news-room/fact-sheets/detail/envejecimiento-y-salud >. Acesso em: 8 ago 2018.

PICARDI, Pablo. Salud Emocional. PÉREZ-LIZAUR, A. B. (Org.). . 10 Hábitos Efectivos para la Salud del Adulto Mayor. 1. ed. Mexico: Universidad Iberoamericana, Instituto Nacional de Ciencias Médicas y Nutrición Salvador Zubirán, Instituto de Geriatría, Instituto Nacional de las Personas Adultas Mayores, 2009. p. 7-11.

SARACCHINI, Rafael e CATALINA, Carlos e BORDONI, Luca. Tecnología asistencial móvil, con realidad aumentada, para las personas mayores. Revista Científica de Educomunicación, v. 23, n. 45, p. 65-74, 2015. Disponível em: $<$ https://www.revistacomunicar.com/index.php?contenido=detalles\&numero $=45 \&$ articulo $=4$ $5-2015-07>$.

\section{Sobre los autores}

\section{Georgina Durán Quezada}

Académica de tiempo completo del Departamento de Diseño de la Universidad Iberoamericana, del cual fue Directora, además de coordinar los programas de Diseño Gráfico y la Maestría en Diseño Estratégico e Innovación. Es Doctora en Diseño por parte de la Pontificia Universidad Católica de Rio de Janeiro, cuyo proyecto exploró la relación entre los adultos mayores y las 
nuevas tecnologías. Actualmente es parte de un grupo de investigación multidisciplinar que realiza propuestas para promover la inclusión infantil.

georgina.duran@ibero.mx

\section{Vera Maria Marsicano Damazio}

Profesora e investigadora en el Departamento de Arte y Diseño de la Pontificia Universidad Católica de Río de Janeiro - PUC-Río (Brasil) desde 1987, donde imparte cursos y trabaja estrechamente con estudiantes graduados y no graduados.

También fundó (1999) y coordina el Laboratorio de Investigación de Diseño, Memoria y Emoción (labmemo), un grupo de investigación interdisciplinario dedicado al desarrollo de productos que se centran en promover una conducta social responsable y el bien colectivo. Organizó el libro "Design Ergonomia Emoção", uno de los primeros libros sobre "diseño emocional" publicado en Brasil (2006).

Doctorado en Ciencias Sociales (Universidad Estatal de Río de Janeiro), Máster en Diseño Gráfico (Boston University) y Licenciado en Diseño Industrial y Comunicación Visual (PUCRio).

vdamazio@puc-rio.br 